\title{
A review of monitoring methods for triclosan and its occurrence in aquatic environments
}

\author{
Hanieh Montaseri ${ }^{1}$, Patricia B.C. Forbes ${ }^{1 *}$ \\ ${ }^{1}$ Department of Chemistry, Faculty of Natural and Agricultural Sciences, University of \\ Pretoria, Lynnwood Road, Pretoria 0002, South Africa
}

\section{HIGHLIGHTS:}

- Triclosan is a widely used biocide which has been detected globally in surface waters.

- Methods for the analysis of triclosan in water samples are reviewed.

- Linear ranges and detection limits of methods based on chromatography-mass spectrometry, electrochemistry, capillary zone electrophoresis and spectrophotometry are compared.

\begin{abstract}
Triclosan is a phenyl ether with broad spectrum antimicrobial action which is employed in a great number of everyday household and personal care products including plastics, fabrics, soaps, deodorants, toothpaste, and cosmetics. There is serious concern, however, regarding this widespread use in terms of the potential environmental impacts of triclosan. Triclosan may enter the aquatic environment via numerous pathways including discharge of effluents from industries and wastewater treatment plants. To date, however, a comprehensive review of the determination of triclosan in aquatic environments has been not reported. Herein, we review the environmental concentration of triclosan in aquatic systems globally, as well as its stability and toxicity. The wide variety of monitoring methods utilized for the determination of triclosan are discussed, including those based on chromatography-mass spectrometry, electrochemistry, capillary zone electrophoresis and spectrophotometry over the last 10 years.
\end{abstract}

\section{Keywords:}

Triclosan

Personal care products 
Electrochemistry

Capillary zone electrophoresis

Molecularly imprinted polymers

Chromatography-mass spectrometry

Spectrophotometry

Chemiluminescence

Abbreviations: APTES, 3-Aminopropyltriethoxysilane; AQA, Analytical Quality Assurance; AuNPs, Gold Nanoparticles; $\beta$-CD/GNP, $\beta$-Clyclodextrin/Graphene Nano Platelets; BTI, Biomarker Response Index; CE, Capillary Electrophoresis; CL, Chemiluminescence; CNPPDDAC, Carbon Nanoparticles-Poly(Dially Dimethyl Ammonium Chloride); CNTs@ $\mathrm{SiO}_{2}$, Carbon Nanotubes Coated with Silica; CNTs@TCS-MIPs, Carbon Nanotubes@Triclosan Molecularly Imprinted Polymer; CTAB, Cetyltrimethylammonium Bromide; CV, Cyclic Voltammetry; CZE-DAD, Capillary Zone Electrophoresis - Diode Array Detection; CZE-UV, Capillary Zone Electrophoresis with UV detection; 2,4-DCP, 2,4-Dichlorophenol; DLLME, Dispersive Liquid-Liquid Microextraction; DLLME-SFO, Dispersive Liquid-Liquid Microextraction Based on Solidification of Floating Organic Droplet; DMBUA, 2,3-dimethyl-6(undec-10-enamide)-6-deoxy- $\beta$-cyclodextrin; DPV, Differential Pulse Voltammetry; DVB/CAR/PDMS, Divinylbenzene/Carboxen/Polydimethylsiloxane; DW, Dry Weight; ECOSAR, Ecological Structure-Activity Relationships; EDCs, Endocrine Disrupting Chemicals; EPA, Environmental Protection Agency;; GAC, Green Analytical Chemistry; GC/ECNI/MS, Gas Chromatography/Electron Capture Negative Ionization/Mass Spectrometry; GC-MS/MS, Gas Chromatography-Tandem Mass Spectrometry; HPLC-ESI-MS/MS, High Performance Liquid Chromatography-Electron Spray Ionization-Tandem Mass Spectrometry; HPLC-UV, High Performance Liquid Chromatography- Ultraviolet Detection; $I_{50}$, The inhibiting concentration at which a reduction of $50 \%$ in survival or reproduction was observed; IL-DLPME, Ionic Liquid Dispersive Liquid Phase Microextraction; IPR, Initial Precision and Recovery; IT-USA-SI-LLME, In-Tube based Ultra-Sound-Assisted Salt-Induced Liquid-Liquid Microextraction; $\mathrm{K}_{\mathrm{oc}}$, Soil Organic Carbon-Water Partitioning Coefficient; LC-DAD, Liquid Chromatography-Diode Array Detection; LC-ESI-MS/MS, Liquid Chromatography-Electron Spray Ionization- Tandem Mass Spectrometry; LC-MS/MS, Liquid Chromatography-Tandem Mass Spectrometry; LVI-GC-MS, Large Volume Injection-Gas Chromatography-Mass Spectrometry; MALLE, MembraneAssisted Liquid-Liquid Extraction; MEPS, Microextraction by Packed Sorbent; MIC, Minimum Inhibitory Concentration; MIP, Molecularly Imprinted Polymer; MWCNTs, Multiwall Carbon Nanotubes; NOEC, No-Observed-Effects Concentration; nZnO-MWCNT/GCE, Nano-Zinc Oxide-Multiwalled Carbon Nanotube/Glassy Carbon Electrode; o-PD/GCE, o-Phenylene Diamine/Glassy Carbon Electrode; OPR, Ongoing Precision and Recovery standard; PA, Polyacrylate; PBDEs, Poly Brominated Diphenyl Ethers; PBT, Persistence, Bioaccumulation and Ecotoxicity; PDDAC, Poly-(Diallyl Dimethyl Ammonium Chloride); PDMS-DVB, Poly (dimethylsiloxane-divinylbenzene); $\quad$ p(HEMAGA), Poly(2-HydroxyethylmethacrylateMethacryloylamidoglutamic Acid); PLE, Pressurized Liquid Extraction; POM, Polyoxometalate; PPCPs, Pharmaceutical and Personal Care Products; QA/QC, Quality Assurance/Quality Control; RDSE, Rotating Disk Sorptive Extraction; rGO, Reduced Graphene Oxide; RP-HPLC, Reverse Phase-High Performance Liquid Chromatography; RSD, Relative Standard Deviation; 
(RSD)SBSE, Stir Bar Sorptive Extraction; SBSE-LD, Stir Bar Sorptive Extraction and Liquid Desorption; SPCEs, Screen-Printed Carbon Electrodes; SPME, Solid Phase Microextraction; SPR, Surface Plasmon Resonance; SWV, Square Wave Voltammetry; 2,4,6-TCP, 2,4,6Trichlorophenol; TCS, Triclosan; TD-GC-MS, Thermal Desorption-Gas Chromatography-Mass Spectrometry; TEOS, Tetraethoxysilicane; TIL-DLME, Temperature-Controlled Ionic LiquidDispersive Liquid Phase Microextraction; UHPLC, Ultra-High-Pressure Liquid Chromatography; UHPLC-TUV, Ultra-High Pressure Liquid Chromatography-Tunable Ultraviolet Detection; UPLC, Ultra Performance Liquid Chromatography; USAEME, Ultrasound-Assisted EmulsificationMicroextraction; USFDA, US Food and Drug Agency; VER, Calibration Verification; WLRS, White Light Reflectance Spectroscopy; WWTPs, Wastewater Treatment Plants

${ }^{*}$ Corresponding author. Tel.:+27 124205426

E-mail address: patricia.forbes@up.ac.za (P.B.C. Forbes). 


\section{Introduction}

Triclosan, or 5-chloro-2-(2,4-dichlorophenoxy) phenol (Figure 1), with the commercial name Irgasan DP300, is a white powder with a slight phenolic odor which has been used in a variety of consumer products [1, 2]. It is an anti-microbial and preservative agent used in personal care products (such as toothpaste, detergent, soap, shampoos, skin care creams and lotions) with a typical concentration in the range of $0.1-0.3 \%$ of product weight. The content of triclosan (TCS) should not exceed $0.3 \%$ (w/w) which is regulated by the European Community Cosmetic Directive or the US Food and Drug Agency (USFDA) in Europe and the USA, respectively [3, 4]

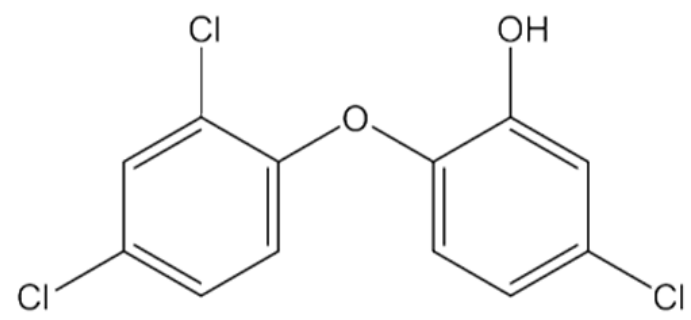

Figure 1 Chemical structure of triclosan.

The solubility of triclosan in water is $<10^{-6} \mathrm{~g} \mathrm{~mL}^{-1}$, although its solubility increases when the $\mathrm{pH}$ becomes more alkaline [5]. The partition coefficient of triclosan ( $\left.\log P_{o w}=5.4\right)$, suggests that it is lipophilic [6].

The halogenated biphenyl ether structure of triclosan is similar to that of bisphenol A, dioxins, polybrominated diphenyl ethers (PBDEs) and thyroid hormones, therefore concern has been raised over triclosan as a potential endocrine disruptor, specifically regarding disruption of thyroid hormone homeostasis $[7,8]$.

The ubiquitous use of triclosan has resulted in its release into wastewater and many water sources [9] where it may affect ecosystems and human health. In particular, triclosan can cause death of algae with a reported $\mathrm{IC}_{50}$ (the inhibiting concentration at which a reduction of $50 \%$ in survival or reproduction was observed) of $4700 \mathrm{ng} \mathrm{L}^{-1}[10]$. There have also been several other health concerns attributed to triclosan, as it can accumulate in the human body over time and may result in long-term health risks [11]. In addition, toxic and persistent compounds such as methyl triclosan, chlorinated phenols and biphenyl ethers may be formed after biological methylation or chlorination of triclosan [12-14]. Some surveys have revealed that methyl triclosan is more lipophilic than its parent compound, contributing to its persistence and bioaccumulation potential in wildlife and humans $[15,16]$. 


\subsection{Concentrations of triclosan in water resources}

Several recent studies have clearly demonstrated the widespread presence of triclosan in the environment, especially in wastewater, wastewater treatment plant effluents, rivers and in sediments in various countries.

A study in the United States identified triclosan as one of the top seven contaminants in surface water, with a maximum concentration of $2300 \mathrm{ng} \mathrm{L}^{-1}$ [17]. The worldwide concentration range of triclosan in water has been found to range from $1.4-40000 \mathrm{ng} \mathrm{L}^{-1}$ in surface waters, $20-86161$ $\mathrm{ng} \mathrm{L}^{-1}$ in wastewater influent, $23-5370 \mathrm{ng} \mathrm{L}^{-1}$ wastewater effluent, $<0.001-100 \mathrm{ng} \mathrm{L}^{-1}$ in sea water, sediment (lake/river/other surface water) $<100-53,000 \mu \mathrm{g} \cdot \mathrm{kg}^{-1}$ dry weight $(\mathrm{dw})$; sediment (marine) $0.02-35 \mu \mathrm{g} \cdot \mathrm{kg}^{-1} \mathrm{dw}$, biosolids from WWTP $20-133,000 \mu \mathrm{g} \cdot \mathrm{kg}^{-1} \mathrm{dw}$, activated/digested sludge $580-15,600 \mu \mathrm{g} \cdot \mathrm{kg}^{-1} \mathrm{dw}$; and pore water $0.201-328.8 \mu \mathrm{g} \cdot \mathrm{L}^{-1}[18,19]$.

Triclosan is a frequent contaminant of aquatic and terrestrial environments and it is detected at a concentration ranging from parts-per-trillion in surface water to parts-per-million in biosolids. The elevated concentration of triclosan in biosolids and aquatic sediments may be attributed to the high usage, strong sorption to organic matter and environmental persistence of this compound [20]. Regarding the half-life, this depends on the environmental compartment and prevailing conditions [21].

Fish, algae, and crustaceans may be affected by elevated levels of biocides in surface waters, the concentrations of which are influenced by raw and treated sewage. It is important to note that triclosan has been detected in surface waters at levels higher than the no-observed-effects concentration (NOEC) of algae [20]. Microorganisms are generally not as sensitive to triclosan compared to non-target species, and the minimum inhibitory concentration (MIC) threshold values for microbes may be increased by the environmental levels of triclosan [20].

A study examined the toxicity ranking of five pharmaceutical and personal care products (PPCPs) including triclosan. Bivalve Dreissena polymorpha in fresh water were exposed to different environmentally relevant concentrations of the PPCPs over $96 \mathrm{hrs}$ and the biological response of eight biomarkers was integrated into a simple biomarker response index (BRI). It was found that triclosan demonstrated dramatic effects. The relative toxicities of the compounds were found to be triclosan $>$ trimethoprim $>$ ibuprofen $>$ diclofenac $=$ paracetamol [22] .

Researchers in Spain conducted another environment risk assessment for 26 PPCPs [23]. They employed Microtox acute ecotoxicity tests and activated sludge respirometry assays. The US EPA Ecological Structure-Activity Relationships (ECOSAR ${ }^{\mathrm{TM}}$ ) QSAR program was also utilized to predict the estimated ecotoxicological effects. Based on the results of ecotoxicity tests, 
triclosan is very toxic to different species. Furthermore, evaluation of persistence, bioaccumulation and ecotoxicity (PBT) displayed that triclosan is persistent and toxic but not bioaccumulative [24].

\subsection{Stability of triclosan in the environment}

The US EPA has reported that triclosan is hydrolytically stable under abiotic and buffered conditions over the $\mathrm{pH}$ range of $4-9$. It degrades with calculated non-linear half-lives of $1.3-$ 1.4 days in water, 53.7 - 60.3 days in sediment, and 39.8 - 55.9 days in aerobic water-sediment systems maintained in darkness, whilst the calculated aqueous photolytic half-life is $41 \mathrm{~min}$ [19, 25].

Triclosan has a $\mathrm{pK}_{a}$ of 7.9 [26] which means that it exists partially in the dissociated form in the environment, which is of relevance as anions do not generally adsorb as strongly to organic carbon and clay as their neutral counterparts [27], although an estimated $\mathrm{K}_{\mathrm{oc}}$ (Soil Organic Carbon-Water Partitioning Coefficient) value of 9,200 has been reported [28]. In aquatic environments, triclosan is expected to adsorb to suspended solids and sediments ( $\mathrm{K}_{\mathrm{ow}} 4.76$ ) $[28,29]$, posing a potential threat to aquatic organisms. Triclosan has an estimated Henry's Law constant of $1.5 \times 10^{-7} \mathrm{~atm} . \mathrm{m}^{3} / \mathrm{mol}[19,29]$, thus it is not anticipated to volatilize readily from soil or water surfaces.

\subsection{Removal of triclosan via wastewater treatment processes}

Triclosan is susceptible to oxidative degradation by ozone and chlorine in the presence of sunlight, and to biodegradation by microorganisms [19]. It has been found that biodegradation is an efficient mechanism for triclosan removal from wastewater and also that biodegradation under aerobic conditions provides higher removal efficiency in comparison with anaerobic media [30].

Wastewater treatment achieves average triclosan removal efficiencies in the range of $58-99 \%$ [31], depending on the technical capabilities of the sewage treatment systems [30, 32-39]. Approximately $50 \%$ of the incoming mass of triclosan, which is produced by activated sludge treatment together with aerobic biosolid digestion in conventional WWTPs, persists and becomes sequestered in biosolids. As a result, major pathways of biocide release into the environment are WWTP effluent discharge into surface waters and the application of biosolids to land [20]. 
Cleavage of the ether bond and chlorination of the phenolic bond were identified as the main degradation pathways for triclosan during wastewater treatment [40]. Five main products were found in the reaction of triclosan with free chlorine including 2,4-dichlorophenol (2,4-DCP), 2,4,6-trichlorophenol (2,4,6-TCP) and tetra- and penta-chlorinated species. 2,4,6-TCP is a known endocrine disruptor, and may cause cancer, birth defects and developmental disorders in offspring, whilst 2,4-DCP may be fatal if large amounts are absorbed by the body [40].

Treatment with ozone during municipal sewage treatment was efficient in the removal of triclosan [41-43]. Although chloramines can be employed, chlorine is a stronger oxidant and has been shown to be more effective at oxidizing pharmaceuticals and endocrine disrupting chemicals (EDCs) in a comparative study where phenolic compounds including triclosan exhibited greater than $95 \%$ removal by chlorination under the conditions tested [44]. The reaction mechanisms of phenolic compounds with free chlorine proceeds via an electrophilic substitution pathway, with a mixture of substituted products at the ortho and para positions. Further chlorine addition results in cleavage of the aromatic ring [44, 45]. It is noteworthy to mention that phototransformation also can remove triclosan from wastewaters [46].

Based on the widespread use of triclosan, its known presence in aquatic systems, as well as the potential environmental impacts it can incur, it is crucial that analytical methods for triclosan are sensitive and robust to allow for the reliable detection and quantification thereof in water systems. Moreover, quality assurance/quality control is needed to ensure reliable analytical information and green analytical chemistry approaches should be considered.

This review discusses the cycling and concentration of triclosan in different environmental compartments and focuses on the development of analytical methods for the determination of triclosan over the last 10 years $(2006-2016)$. It should be noted that although some of the methods discussed have been developed to analyse a class of pharmaceutical compounds, we have limited our discussion to triclosan in aquatic media. Reported analytical methods in this regard are thus reviewed in this paper to assist readers in the evaluation and comparison of the different options which have been tested.

\section{Green analytical chemistry}

The term "green analytical chemistry (GAC)" was proposed by J. Namieśnik [47] and has gained a great deal of attention in recent years. The main purposes of GAC are not only making laboratory practices more environmentally friendly, but also reducing the negative impact of chemical analyses on the environment and to enable implementation of sustainable 
development principles in analytical laboratories. One of the most important challenges for the future of GAC is to provide a compromise between increasing the quality of the results in terms of selectivity, sensitivity, reliability of analysis and reducing analysis time on one hand, and improving environmental friendliness of analytical methods on the other. In 2013, Namieśnik et al proposed the following 12 principles of GAC [48]:

1. Direct analytical techniques should be applied to avoid sample treatment.

2. Minimal sample size and minimal number of samples are goals.

3. In situ measurements should be performed.

4. Integration of analytical processes and operations to save energy and reduce the use of reagents.

5. Automated and miniaturized methods should be selected.

6. Derivatization should be avoided.

7. Generation of a large volume of analytical waste should be avoided and proper management of analytical waste should be provided.

8. Multi-analyte or multi-parameter methods are preferred versus methods for one analyte at a time.

9. The use of energy should be minimized.

10. Reagents obtained from renewable sources should be preferred.

11. Toxic reagents should be eliminated or replaced.

12. The safety of the operator should be increased.

In this way, the principles of green chemistry can be applied to the analytical field. However, a number of the 12 principles may cause a decrease in analytical performance parameters such as accuracy, precision and sensitivity.

Table 1 compares less green techniques used for determination of triclosan with their greener alternatives. 
Table 1. Examples of green techniques compared to conventional analytical methods and their relative advantages and disadvantages.

\begin{tabular}{|c|c|c|c|}
\hline \multicolumn{4}{|c|}{ Less green analytical methods } \\
\hline Analytical method & Potential advantages & Potential disadvantages & References \\
\hline HPLC & $\begin{array}{c}\text { Rapid, sensitive, accurate } \\
\text { and selective, small sample } \\
\text { volume, }\end{array}$ & $\begin{array}{c}\text { Potentially poor repeatability, } \\
\text { consumes reagents, produces } \\
\text { wastes }\end{array}$ & {$[49,50]$} \\
\hline GC-MS & $\begin{array}{l}\text { Low detection limit, selective, } \\
\text { sensitive, accurate, low } \\
\text { sample volume }\end{array}$ & $\begin{array}{c}\text { Requires the use of toxic } \\
\text { reagents and derivatization, } \\
\text { consumes reagents, produces } \\
\text { wastes, time-consuming, multi- } \\
\text { stage sample treatment often } \\
\text { necessary }\end{array}$ & {$[51,52]$} \\
\hline Spectrophotometry & Simple, rapid, inexpensive & $\begin{array}{l}\text { High detection limit, consumes } \\
\text { reagents, produces wastes, poor } \\
\text { sensitivity and selectivity, uses } \\
\text { toxic and corrosive reagents }\end{array}$ & {$[53,54]$} \\
\hline \multicolumn{4}{|c|}{ Greener methods } \\
\hline $\begin{array}{l}\text { Electrochemical } \\
\text { sensor }\end{array}$ & $\begin{array}{l}\text { Simple and fast, low detection } \\
\text { limits, sensitive, simple, rapid, } \\
\text { reliable, inexpensive, low } \\
\text { energy consumption }\end{array}$ & $\begin{array}{l}\text { Sometimes poor reproducibility, } \\
\text { needs monitoring of electrode } \\
\text { stability, possible interferences } \\
\text { with other analytes }\end{array}$ & [55-57] \\
\hline Electrophoresis & $\begin{array}{l}\text { Small sample volume, } \\
\text { reduced reagent volumes, } \\
\text { rapid, portable, uses little } \\
\text { power }\end{array}$ & $\begin{array}{l}\text { Often poor reproducibility and } \\
\text { sensitivity }\end{array}$ & {$[58,59]$} \\
\hline
\end{tabular}


Selection of the greenest method from all available methods is based on the needs of the user such as accuracy, selectivity and detection limits. Typically direct, automated, miniaturized and portable analytical techniques are the greenest options [60]. In addition, multi-analyte or multiparameter methods are of great importance for environmental monitoring, where information on a number of analytes can be obtained from a single analysis [48]. The majority of reagents to be replaced in analytical procedures are organic solvents [60] where ionic liquids and supercritical fluids are considered as alternative (green) solvents. These are finding application in chromatography, electrochemistry and spectroscopy [61]. Green aspects of the analytical methods discussed in this paper are provided to assist readers in evaluating the different options available and the areas where further improvements in this regard should be investigated.

\section{Quality assurance/quality control}

Green analytical chemistry entails performing reliable analysis while applying quality assurance/quality control (QA/QC) and simultaneously minimizing any environmental impacts resulting from the analysis [62]. The minimum requirements for $Q A / Q C$ comprise of an initial demonstration of laboratory capability, analysis of samples spiked with labeled compounds to evaluate data quality, and analysis of standards and blanks as tests of continued performance [63]. QA activities may include participation in laboratory accreditation programs, external system audits, and inter-laboratory comparisons [64]. Analytical method validation forms the first level of QA in the laboratory. Moreover, analytical quality assurance (AQA) is the complete set of measures a laboratory must undertake to ensure that it can always achieve high-quality data [65].

The US EPA conducted research regarding the determination of pharmaceuticals and personal care products in water, soil, sediment and biosolids by HPLC-MS/MS [63]. Based on this study, relative standard deviation (RSD) and the average percent recovery $(X)$ with the corresponding limits for each native and labeled compound were compared in order to investigate initial precision and recovery (IPR). The requirement is that if RSD and $X$ for all compounds meet the acceptance criteria, system performance is acceptable and analysis of blanks and samples may begin. If, however, any individual RSD exceeds the precision limit or any individual $X$ falls outside the range for recovery, system performance is unacceptable for that compound and requires correction. In addition, the recovery of each labeled compound must be within the limits 
to ensure method performance. If the recovery of any compound falls outside of these limits, additional cleanup procedures may be employed or the sample size should be adjusted. Proposed QC parameters for triclosan are given in Table 2 [63].

Table 2. Proposed QC acceptance criteria for triclosan [63].

\begin{tabular}{|c|c|c|c|c|c|}
\hline \multirow{2}{*}{ Compound } & \multirow{2}{*}{$\begin{array}{c}\text { (\%) Calibration } \\
\text { Verification } \\
\text { (VER) }\end{array}$} & \multicolumn{2}{|c|}{$\begin{array}{c}\text { Initial Precision and } \\
\text { Recovery (IPR) }\end{array}$} & $\begin{array}{c}\text { Labeled } \\
\text { Ongoing } \\
\text { Precision and } \\
\text { Recovery } \\
\text { standard (OPR) }\end{array}$ & $\begin{array}{c}\text { compound } \\
\text { recovery in } \\
\text { samples (\%) }\end{array}$ \\
Triclosan & $70-130$ & 30 & $55-108$ & $50-120$ & \\
\hline${ }^{13} \mathrm{C}_{12}$-Triclosan & $70-130$ & 30 & $6-151$ & $6-168$ & $5-153$ \\
\hline
\end{tabular}

\section{Monitoring methods for triclosan}

A range of techniques have been employed in the monitoring of triclosan in different matrices. Although not all of the methods discussed in this section were developed specifically for the monitoring of triclosan in water samples, they have the potential to be used for this application. In addition, it should be noted that some methods are for the simultaneous analysis of a range of pharmaceutical compounds, as indicated in Table 3.

\subsection{Chromatography-Mass Spectrometry}

Numerous chromatography methods coupled with mass spectrometry have been employed to selectively detect low levels of triclosan in aqueous systems, but these methods usually have disadvantages in terms of analytical running costs, complexity, environmentally unfriendly solvent use, expensive instrumentation, long analysis time, as well as the need for highly skilled technicians and laborious sample pre-treatment [56, 66]. Rapid and sensitive screening methods may, therefore, provide desirable alternatives, as discussed in later sections of this paper. Table 3 shows a range of chromatography-based methods for triclosan (and in some cases additional pharmaceutical analytes) and compares the linear ranges and detection limits 
for triclosan. The sample extraction (pre-concentration) technique, derivatization reagent employed, and the detector employed are also included.

Table 3. Comparison of selected chromatography-based methods for triclosan determinations in water samples.

\begin{tabular}{|c|c|c|c|c|c|c|c|c|}
\hline Method & $\begin{array}{l}\text { Extraction } \\
\text { method }\end{array}$ & Analytes & $\begin{array}{l}\text { Derivatization } \\
\text { reagent }\end{array}$ & $\begin{array}{l}\text { Linear } \\
\underset{1}{\text { range }}\left(\mathrm{ng} \mathrm{L}^{-}\right.\end{array}$ & $\underset{\left.L^{-1}\right)}{\operatorname{LOD}}(n g$ & LOQ $\left(n g L^{-1}\right)$ & Samples & Reference \\
\hline GC-MS & $\begin{array}{l}\text { SPE (GFF glass } \\
\text { fiber filter) }\end{array}$ & $\begin{array}{c}\text { Triclosan, } \\
\text { Nonylphenol } \\
\text {, } \\
\text { Nonylphenol } \\
\text { ethoxylates, } \\
\text { and } \\
\text { Bisphenol A }\end{array}$ & $\begin{array}{l}\text { Bis(trimethylsil } \\
\text { yl)trifluoroaceta } \\
\text { mide }\end{array}$ & $\begin{array}{c}1.0 \times 10^{6}- \\
1.0 \times 10^{7}\end{array}$ & 130.3 & 434.3 & Wastewater & [67] \\
\hline GC-MS & $\begin{array}{l}\text { Oasis HLB } \\
\text { cartridge }\end{array}$ & $\begin{array}{c}\text { Triclosan, } \\
\text { Acidic } \\
\text { pharmaceuti } \\
\text { cals, } \\
\text { Phenols, } \\
\text { Estrone, } \\
17 \beta- \\
\text { estradiol, } \\
17 \alpha- \\
\text { ethinylestrad } \\
\text { iol }\end{array}$ & $\begin{array}{l}\text { MTBSTFA, } \\
\text { TBDMSCI, } \\
\text { BSTFA, } \\
\text { TMSCI }\end{array}$ & $18.0-2.3 \times 10^{4}$ & 10.1 & - & $\begin{array}{c}\text { Untreated } \\
\text { wastewater }\end{array}$ & [51] \\
\hline GC-MS & SBSE-LD & $\begin{array}{l}\text { Triclosan, } \\
\text { Methyl } \\
\text { triclosan }\end{array}$ & MTBSTFA & $20.0-2.0 \times 10^{4}$ & 5.0 & - & River water & [68] \\
\hline GC-MS & RDSE & $\begin{array}{l}\text { Triclosan, } \\
\text { Methyl } \\
\text { triclosan }\end{array}$ & MTBSTFA & $\begin{array}{l}160.0- \\
2000.0\end{array}$ & 46.0 & 152.0 & $\begin{array}{l}\text { Untreated } \\
\text { wastewater }\end{array}$ & [69] \\
\hline \multirow[b]{2}{*}{ GC-MS } & \multirow{2}{*}{$\begin{array}{l}\text { Oasis HLB } \\
\text { cartridge }\end{array}$} & \multirow{2}{*}{$\begin{array}{l}\text { Triclosan, } \\
\text { Pharmaceuti } \\
\text { cals and } \\
\text { Endocrine- } \\
\text { disrupting } \\
\text { substances }\end{array}$} & \multirow[b]{2}{*}{ MTBSTFA } & \multirow[b]{2}{*}{$5.0-202.7$} & $<58.0$ & \multirow[b]{2}{*}{-} & $\begin{array}{c}\text { Drinking } \\
\text { water }\end{array}$ & \multirow[b]{2}{*}{ [52] } \\
\hline & & & & & $<101.3$ & & $\begin{array}{l}\text { Surface } \\
\text { water }\end{array}$ & \\
\hline GC-MS & \multirow{2}{*}{$\begin{array}{c}\text { In-drop } \\
\text { derivatisation } \\
\text { liquid-phase } \\
\text { microextraction }\end{array}$} & \multirow{2}{*}{$\begin{array}{l}\text { Triclosan, } 9 \\
\text { known } \\
\text { phenolic } \\
\text { endocrine } \\
\text { disruptors }\end{array}$} & \multirow{2}{*}{$\begin{array}{c}\text { Ethyl } \\
\text { chloroformate }\end{array}$} & $1100-28 \times 10^{3}$ & 400 & \multirow{2}{*}{ - } & \multirow{2}{*}{$\begin{array}{c}\text { Surface } \\
\text { water, } \\
\text { wastewater }\end{array}$} & \multirow{2}{*}{ [70] } \\
\hline GC-FID & & & & $\begin{array}{l}55 \times 10^{3} \\
72 \times 10^{4}\end{array}$ & 18300 & & & \\
\hline TD-GC-MS & SBSE (PDMS) & Triclosan & NA & $20.0-2.0 \times 10^{4}$ & 5.0 & 20.0 & River water & [71] \\
\hline
\end{tabular}




\begin{tabular}{|c|c|c|c|c|c|c|c|c|}
\hline TD-GC-MS & SBSE & $\begin{array}{l}\text { Triclosan, } \\
\text { Methyl } \\
\text { triclosan, } \\
\text { Paraben }\end{array}$ & $\begin{array}{c}\text { Acetic } \\
\text { anhydride in } \\
\text { basic medium }\end{array}$ & - & 1.39 & 4.65 & $\begin{array}{l}\text { Tap water, } \\
\text { untreated } \\
\text { and treated } \\
\text { wastewater }\end{array}$ & [72] \\
\hline \multirow{2}{*}{ LVI-GC-MS } & \multirow{2}{*}{ MEPS } & \multirow{2}{*}{$\begin{array}{l}\text { Triclosan, } \\
\text { Methyl } \\
\text { triclosan, } \\
\text { Paraben }\end{array}$} & \multirow{2}{*}{ NA } & \multirow{2}{*}{$\begin{array}{c}100.0- \\
1.0 \times 10^{4}\end{array}$} & $1.0-15.0$ & 3.3-49.5 & $\begin{array}{l}\text { Ultrapure } \\
\text { water }\end{array}$ & \multirow{2}{*}{ [73] } \\
\hline & & & & & $\begin{array}{l}20.0- \\
590.0\end{array}$ & $66.0-2 \times 10^{3}$ & $\begin{array}{c}\text { Raw } \\
\text { wastewater }\end{array}$ & \\
\hline GC-MS/MS & $\begin{array}{l}\text { C18 SPE } \\
\text { cartridge }\end{array}$ & Triclosan & NA & $145.0-211.4$ & 0.26 & - & $\begin{array}{l}\text { Wastewater } \\
\text {, river, sea } \\
\text { water }\end{array}$ & [74] \\
\hline GC-MS/MS & $\begin{array}{l}\text { Oasis HLB } \\
\text { sorbent }\end{array}$ & $\begin{array}{l}\text { Triclosan, } \\
\text { Neutral and } \\
\text { acidic } \\
\text { pharmaceuti } \\
\text { cals }\end{array}$ & NA & $9.8-984.4$ & 2.0 & 8.7 & $\begin{array}{l}\text { Hospital } \\
\text { effluent }\end{array}$ & [75] \\
\hline GC-MS/MS & $\begin{array}{l}\text { SPE (C18 } \\
\text { cartridge) }\end{array}$ & Triclosan & NA & $4.1-115.8$ & 20.3 & - & $\begin{array}{l}\text { River and } \\
\text { coastal } \\
\text { water }\end{array}$ & [76] \\
\hline GC-MS/MS & $\begin{array}{c}\text { SPME } \\
\text { (DVB/CAR/PDM } \\
\text { S) }\end{array}$ & $\begin{array}{l}\text { Triclosan, } \\
\text { Paraben, } \\
\text { Related } \\
\text { chlorophenol } \\
\text { s }\end{array}$ & $\begin{array}{c}\text { Acetic } \\
\text { anhydride }\end{array}$ & $10.0-5000$ & 6.5 & 28 & $\begin{array}{c}\text { River } \\
\text { water, } \\
\text { wastewater } \\
\text { s and } \\
\text { swimming } \\
\text { pool water }\end{array}$ & [77] \\
\hline GC-MS/MS & DLLME & $\begin{array}{l}\text { Triclosan, } \\
\text { Methyl } \\
\text { triclosan }\end{array}$ & MTBSTFA & $10.0-2000.0$ & 6.0 & 2.0 & $\begin{array}{l}\text { Tap water, } \\
\text { river water, } \\
\text { treated } \\
\text { wastewater } \\
\text { and raw } \\
\text { wastewater }\end{array}$ & [78] \\
\hline GC-MS/MS & USAEME & $\begin{array}{c}\text { Triclosan, } \\
\text { phenolic } \\
\text { preservative } \\
\text { s }\end{array}$ & $\begin{array}{l}\text { Acetic } \\
\text { anhydride }\end{array}$ & $\begin{array}{l}1.0 \times 10^{3}- \\
1.4 \times 10^{6}\end{array}$ & 5.84 & - & $\begin{array}{l}\text { Influent and } \\
\text { effluent } \\
\text { wastewater } \\
\text { s, river } \\
\text { waters and } \\
\text { swimming } \\
\text { pool water }\end{array}$ & [79] \\
\hline GC-MS/MS & MALLE & $\begin{array}{l}\text { Triclosan, } \\
\text { Paraben }\end{array}$ & $\begin{array}{c}\text { Acetic } \\
\text { anhydride and } \\
\text { Potassium } \\
\text { hydrogenphos } \\
\text { phate }\end{array}$ & $10.0-5000.0$ & 1.1 & 3.6 & $\begin{array}{c}\text { Treated } \\
\text { and raw } \\
\text { wastewater } \\
\text { s }\end{array}$ & [80] \\
\hline
\end{tabular}




\begin{tabular}{|c|c|c|c|c|c|c|c|c|}
\hline RP-LC-DAD & SBSE-LD & Triclosan & NA & $\begin{array}{c}400.0- \\
108.0 \times 10^{3}\end{array}$ & 100.0 & 400.0 & $\begin{array}{c}\text { Urban } \\
\text { wastewater } \\
,\end{array}$ & [81] \\
\hline \multirow{2}{*}{ HPLC-DAD } & \multirow{2}{*}{ SPME (PDMS) } & \multirow{2}{*}{$\begin{array}{l}\text { Triclosan, } \\
\text { Triclocarban, } \\
\text { and } \\
\text { transformati } \\
\text { on products } \\
\text { of } \\
\text { triclocarban }\end{array}$} & \multirow{2}{*}{ NA } & \multirow{2}{*}{$\begin{array}{l}250.0- \\
1.0 \times 10^{5}\end{array}$} & 60.0 & \multirow{2}{*}{ - } & $\begin{array}{l}\text { Deionized } \\
\text { water }\end{array}$ & \multirow{2}{*}{82} \\
\hline & & & & & 470.0 & & River water & \\
\hline HPLC-DAD & SBSE & $\begin{array}{c}\text { Triclosan, 4- } \\
\text { n- } \\
\text { nonylphenol, } \\
\text { and di-n- } \\
\text { butyl } \\
\text { phthalate }\end{array}$ & NA & $\begin{array}{l}1.0 \times 10^{4}- \\
1.0 \times 10^{7}\end{array}$ & - & $1.0 \times 10^{4}$ & $\begin{array}{l}\text { Bottled } \\
\text { water }\end{array}$ & [83] \\
\hline HPLC-UV & $\begin{array}{l}\text { On-line solid- } \\
\text { phase } \\
\text { microextraction }\end{array}$ & $\begin{array}{c}\text { Triclosan, } \\
2,4- \\
\text { dichlorophen } \\
\text { ol, 2,4,6- } \\
\text { trichlorophen } \\
\text { ol, 3- } \\
\text { chlorophenol } \\
\text {, Bisphenol } \\
\text { A, } \\
\text { Mefenamic } \\
\text { acid and } \\
\text { Gemfibrozil }\end{array}$ & NA & $\begin{array}{c}10.0- \\
168.0 \times 10^{3}\end{array}$ & 1.0 & - & $\begin{array}{l}\text { Tap water, } \\
\text { river water } \\
\text { and } \\
\text { municipal } \\
\text { wastewater }\end{array}$ & [49] \\
\hline HPLC-UV & \multirow[b]{2}{*}{ DLLME-SFO } & \multirow{2}{*}{$\begin{array}{l}\text { Triclosan } \\
\text { and 2,4- } \\
\text { dichlorophen } \\
\text { ol }\end{array}$} & \multirow{2}{*}{ NA } & $\begin{array}{c}500.0- \\
5.0 \times 10^{5}\end{array}$ & 100.0 & \multirow{2}{*}{ - } & \multirow{2}{*}{$\begin{array}{l}\text { Tap water, } \\
\text { lake water } \\
\text { and river } \\
\text { water }\end{array}$} & \multirow{2}{*}{ [50] } \\
\hline LC-MS/MS & & & & $20.0-1.0 \times 10^{4}$ & 2.0 & & & \\
\hline \multirow{4}{*}{ LC-MS/MS } & \multirow{4}{*}{$\begin{array}{l}\text { SPE- Strata-X } \\
\text { 33U Polymeric } \\
\text { Reversed } \\
\text { Phase }\end{array}$} & \multirow{4}{*}{$\begin{array}{l}\text { Triclosan } \\
\text { and } 20 \\
\text { acidic } \\
\text { pharmaceuti } \\
\text { cals and } \\
\text { personal } \\
\text { care } \\
\text { products }\end{array}$} & \multirow{4}{*}{ NA } & Ave $=0.0$ & - & 1.0 & $\begin{array}{l}\text { Drinking } \\
\text { water }\end{array}$ & \multirow{4}{*}{ [84] } \\
\hline & & & & Ave $=1.0$ & - & 3.0 & River water & \\
\hline & & & & Ave $=2.3$ & - & 5.0 & $\begin{array}{l}\text { Wastewater } \\
\text { influent }\end{array}$ & \\
\hline & & & & Ave $=46.0$ & - & 5.0 & $\begin{array}{c}\text { Wastewater } \\
\text { effluent }\end{array}$ & \\
\hline & & $\begin{array}{l}\text { Triclosan, } \\
\text { Triclocarban, } \\
\text { and }\end{array}$ & & & 1.28 & 4.22 & $\begin{array}{c}\text { Deionized } \\
\text { water }\end{array}$ & \\
\hline tis Letricivis & SPME (PDMS) & $\begin{array}{l}\text { on products } \\
\text { of } \\
\text { triclocarban }\end{array}$ & 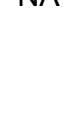 & $0.0-100.0$ & 1.37 & 4.52 & River water & {$[0<]$} \\
\hline
\end{tabular}




\begin{tabular}{|c|c|c|c|c|c|c|c|c|}
\hline $\begin{array}{l}\text { HPLC-ESI- } \\
\text { MS/MS }\end{array}$ & IL-DLPME & $\begin{array}{l}\text { Triclosan } \\
\text { and } \\
\text { Triclocarban }\end{array}$ & NA & $\begin{array}{l}1.0 \times 10^{3}- \\
6.0 \times 10^{4}\end{array}$ & 580.0 & - & $\begin{array}{l}\text { Tap water } \\
\text { and } \\
\text { wastewater }\end{array}$ & [85] \\
\hline $\begin{array}{l}\text { HPLC-ESI- } \\
\text { MS/MS }\end{array}$ & $\begin{array}{c}\text { Temperature- } \\
\text { controlled ionic } \\
\text { liquid dispersive } \\
\text { liquid-phase } \\
\text { microextraction }\end{array}$ & $\begin{array}{l}\text { Triclosan } \\
\text { and } \\
\text { Triclocarban }\end{array}$ & NA & $50.0-1.0 \times 10^{5}$ & $3.0 \times 10^{2}$ & - & $\begin{array}{l}\text { Lake water, } \\
\text { river water, } \\
\text { and tap } \\
\text { water }\end{array}$ & [86] \\
\hline $\begin{array}{l}\text { HPLC-ESI- } \\
\text { MS/MS }\end{array}$ & $\begin{array}{l}\text { lonic liquid/ionic } \\
\text { liquid dispersive } \\
\text { liquid liquid } \\
\text { microextraction }\end{array}$ & $\begin{array}{l}\text { Triclosan } \\
\text { and } \\
\text { Triclocarban }\end{array}$ & NA & $\begin{array}{l}2.5 \times 10^{3}- \\
5.0 \times 10^{5}\end{array}$ & $3.5 \times 10^{2}$ & - & $\begin{array}{c}\text { Tap water, } \\
\text { river water, } \\
\text { snow water } \\
\text { and lake } \\
\text { water }\end{array}$ & [87] \\
\hline HPLC-ESI/MS & $\begin{array}{l}\text { Microporous } \\
\text { bamboo- } \\
\text { activated } \\
\text { charcoal solid- } \\
\text { phase } \\
\text { extraction } \\
\end{array}$ & Triclosan & NA & $20.0-2.0 \times 10^{4}$ & 2.0 & - & $\begin{array}{c}\text { River } \\
\text { waters }\end{array}$ & [88] \\
\hline UHPLC & DLLME & $\begin{array}{l}\text { Triclosan, } \\
\text { Triclocarban, } \\
\text { Methyl- } \\
\text { triclosan }\end{array}$ & NA & $50.0-1.0 \times 10^{5}$ & 134.0 & - & $\begin{array}{c}\text { River } \\
\text { water, } \\
\text { irrigating } \\
\text { water, } \\
\text { reclaimed } \\
\text { water and } \\
\text { domestic } \\
\text { water } \\
\end{array}$ & [66] \\
\hline UHPLC-TUV & TIL-DLME & $\begin{array}{l}\text { Triclosan, } \\
\text { Triclocarban, } \\
\text { Methyl- } \\
\text { triclosan }\end{array}$ & NA & $10.0-1.0 \times 10^{5}$ & 1.15 & - & $\begin{array}{l}\text { Reclaimed } \\
\text { water, } \\
\text { irrigating } \\
\text { water, } \\
\text { wastewater } \\
\text { and } \\
\text { domestic } \\
\text { water }\end{array}$ & [89] \\
\hline $\begin{array}{l}\text { UHPLC- } \\
\text { MS/MS }\end{array}$ & $\begin{array}{l}\text { SPE (Oasis } \\
\text { HLB and Bond } \\
\text { Elut Plexa) }\end{array}$ & $\begin{array}{c}\text { Triclosan, } \\
\text { Triclocarban, } \\
4 \\
\text { preservative } \\
\text { s, } 5 \text { UV } \\
\text { filters }\end{array}$ & NA & $\begin{array}{l}500.0- \\
5.0 \times 10^{5}\end{array}$ & 200.0 & 5.0 & $\begin{array}{c}\text { Surface } \\
\text { and } \\
\text { wastewater } \\
\text { s }\end{array}$ & [90] \\
\hline
\end{tabular}

BSTFA N,O-bis(trimethylsilyl)trifluoroacetamide

DLLME Dispersive Liquid-Liquid Microextraction

DLLME-SFO Dispersive Liquid-Liquid Microextraction Based on Solidification of Floating Organic Droplet

DVB/CAR/PDMS Divinylbenzene/Carboxen/Polydimethylsiloxane

GC/ECNI/MS Gas Chromatography/Electron Capture Negative Ionization/Mass Spectrometry

GC-MS/MS Gas Chromatography - Tandem Mass Spectrometry

HPLC-ESI-MS/MS High Performance Liquid Chromatography-Electron Spray Ionization- Tandem Mass

Spectrometry

HPLC-UV

High Performance Liquid Chromatography- Ultraviolet Detection

IL-DLPME Ionic Liquid - Dispersive Liquid Phase Microextraction

IT-USA-SI-LLME In-Tube based Ultra-Sound-Assisted Salt-Induced Liquid-Liquid Microextraction

LC-DAD Liquid Chromatography - Diode Array Detection

LC-ESI-MS/MS Liquid Chromatography-Electron Spray lonization- Tandem Mass Spectrometry

LC-MS/MS Liquid Chromatography- Tandem Mass Spectrometry 
LVI-GC-MS Large Volume Injection-Gas Chromatography-Mass Spectrometry

MALLE Membrane-Assisted Liquid-Liquid Extraction

MEPS Microextraction by Packed Sorbent

MTBSTFA N-tert-butyldimethylsilyl-N-methyltrifluoroacetamide

NA Not Applicable

PLE Pressurized Liquid Extraction

RDSE Rotating Disk Sorptive Extraction

RP-HPLC Reverse Phase-High Performance Liquid Chromatography

SBSE Stir Bar Sorptive Extraction

SBSE-LD Stir Bar Sorptive Extraction and Liquid Desorption

SPE Solid Phase Extraction

SPME Solid Phase Microextraction

TBDMSCl Tert-butyldimethylsilylchlorane

TD-GC-MS Thermal Desorption-Gas Chromatography-Mass Spectrometry

TIL-DLME Temperature-Controlled Ionic Liquid - Dispersive Liquid Phase Microextraction

$\mathrm{TMSCl}$ Trimethylsilylchlorane

UHPLC Ultra-High-Pressure Liquid Chromatography

UHPLC-TUV Ultra-High Pressure Liquid Chromatography-Tunable Ultraviolet Detection

UPLC Ultra Performance Liquid Chromatography

USAEME Ultrasound-Assisted Emulsification-Microextraction

In addition to the extraction methods listed in Table 3, research has been conducted into the use of molecularly imprinted polymers (MIPs) as extraction media for triclosan. Gao and coworkers, for example, synthesized core-shell MIPs on silica-coated multi-walled carbon nanotubes (MWCNTs) via a sol-gel process for the determination of triclosan in environmental water samples with HPLC analysis (Fig. 2). The binding isotherms of TCS were determined in the concentration range of $3.0 \times 10^{6}-1.2 \times 10^{10} \mathrm{ng} \mathrm{L}^{-1}[91]$.

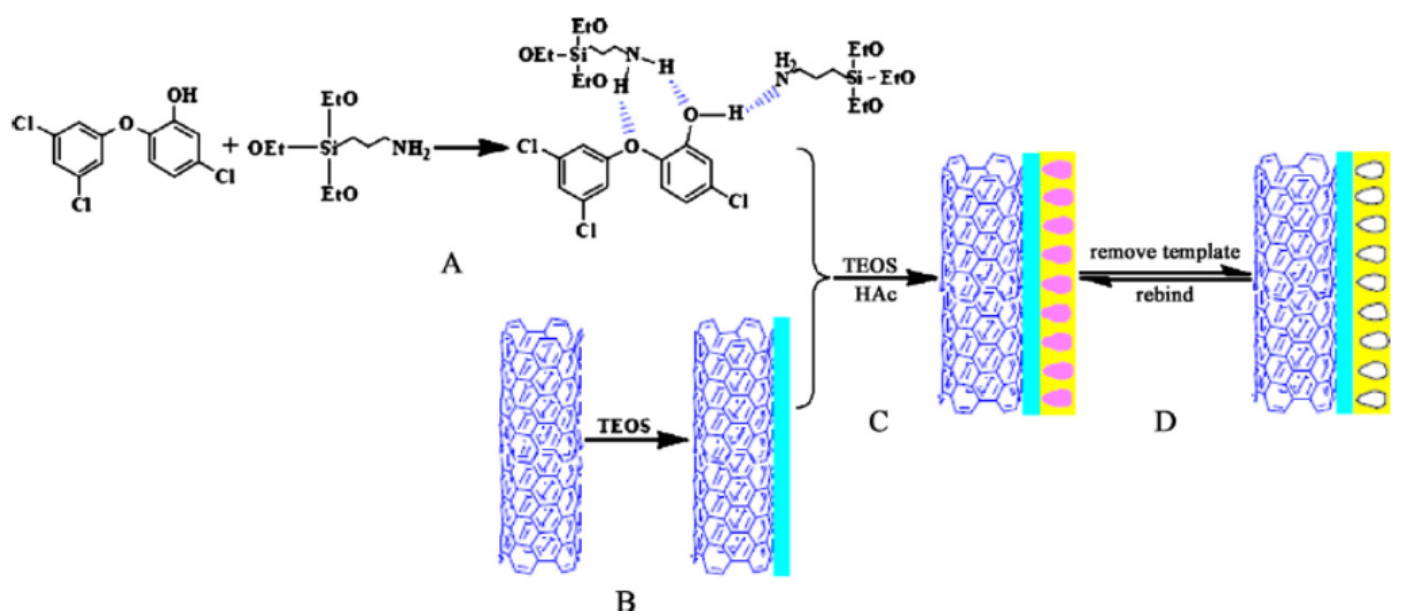

Figure 2 Scheme for the synthesis of carbon nanotubes@triclosan - molecularly imprinted polymer (CNTs@TCSMIPs): (a) formation of template triclosan (TCS)-aminosilica monomer 3-aminopropyltriethoxysilane (APTES) complex; (b) transformation of the surface of purified CNTs to silica shell by a sol-gel process using tetraethoxysilicane (TEOS) and APTES in the presence of cetyltrimethylammonium bromide (CTAB) to obtain core@shell carbon nanotubes coated with silica (CNTs@SiO2); (c) reaction of CNTs@SiO2 with template silica monomer complex to produce silica surface functionalized with TCS-imprinted polymer; (d) removal of the TCS from polymer shells to obtain the CNTs@TCS-MIPs [91]. 
Developments have been observed to make chromatography more environmentally friendly, such as the substitution of organic solvents with less toxic alternatives and there has been a shift towards miniaturization [92].

One of the main advantages of chromatographic procedures is that they facilitate the analysis of hundreds of pollutants in a single run [93], however sample preparation can have the most detrimental effect on the environment. In recent years the development of dispersive liquidliquid microextraction (DLLME); liquid-phase microextraction (LPME), and single drop microextraction (SDME) has enabled greener sample preparation procedures due to the lower solvent volumes employed [94].

It is widely accepted that LC is less "green" than GC because LC mobile phases are usually solvents (unless water is used). One of the most commonly used toxic solvents in LC is acetonitrile thus one of the most common approaches to designing an appropriate green mobile phase in reversed-phase high performance liquid chromatography (RP-HPLC) is to add small volumes of modifiers to water [81].

\subsection{Electrochemical methods}

The application of electrochemical techniques has dramatically increased as a consequence of the development of more sophisticated instrumentation and novel electrode materials. Compared to other analytical techniques, the direct electrochemical analysis of triclosan is simple, rapid, sensitive and cost-effective and is based on the phenolic hydroxyl of triclosan which can undergo oxidation processes under optimal conditions. More importantly this technique enjoys the advantage of a broad linear range [95]. The first direct electrochemical method for triclosan was proposed by Pemberton et al. [26], which employed cyclic voltammetry and screen-printed carbon electrodes (SPCEs) which were disposable, could be mass produced at low cost, and had a wide linear range.

Although the detection of triclosan has been investigated using a mercury electrode [96], it suffers from a narrow linear range and is considered to be an environmentally unfriendly technique. High temperature-high mass transport conditions were later induced by focused microwaves at glassy carbon and boron doped diamond electrode surfaces in order to enhance the electrochemical oxidation of triclosan [97]. In spite of the fact that the hydrophobic nature of triclosan resulted in binding thereof onto the electrode surface, it provided a good detection limit compared to other electrochemical methods and this problem can be overcome by the utilization of surfactants [98]. 
Chemically modified electrodes, such as a composite nano-zinc oxide-multiwalled carbon nanotube modified glassy carbon electrode, have been found to accelerate electron transfer for electro-oxidation of triclosan [99].

A study compared the electrochemical determination of triclosan based on (i) the extraction of triclosan onto carbon nanoparticles followed by deposition onto electrodes and (ii) the adsorption of triclosan directly onto carbon nanoparticles immobilized at electrodes, where the extraction process was found to be highly effective [100].

Table 4 summarizes and compares the linear ranges and detection limits for the detection of triclosan achieved using a range of electrochemical methods. It is evident that in most cases extraction prior to analysis is not required and that typically triclosan alone is determined.

Table 4 Comparison of linear ranges and detection of limits (LODs) for triclosan obtained using a range of electrode based systems.

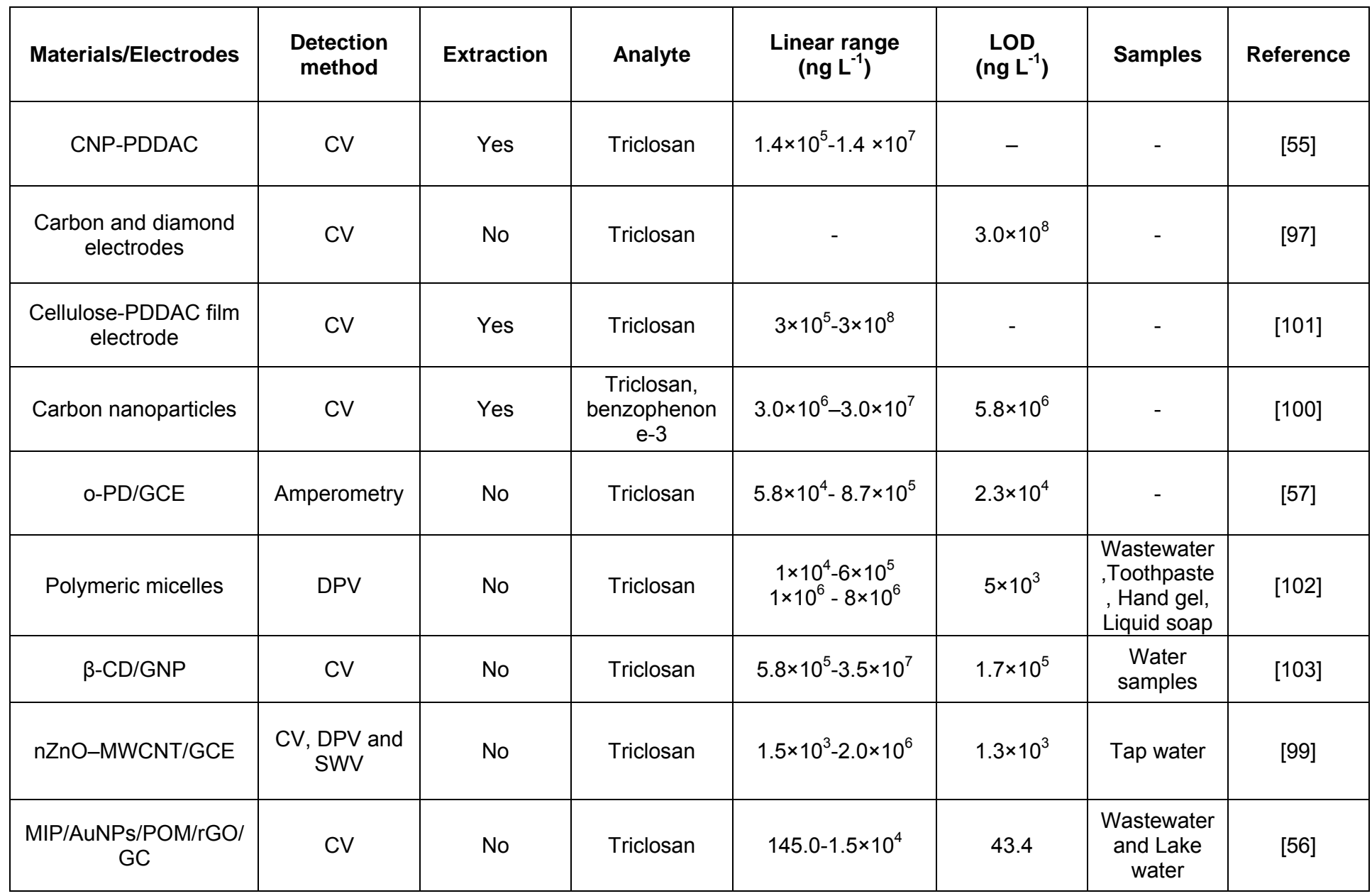

AuNPs Gold Nanoparticles

$\beta$-CD/GNP $\quad \beta$-Clyclodextrin/Graphene Nano Platelets

CNP-PDDAC Carbon Nanoparticles-Poly(Dially Dimethyl Ammonium Chloride) 
CV Cyclic Voltammetry

DPV Differential Pulse Voltammetry

MIP Molecularly Imprinted Polymer

MWCNTs Multiwall carbon Nanotubes

nZnO-MWCNT/GCE Nano-Zinc Oxide-Multiwalled Carbon Nanotube/Glassy Carbon Electrode

o-PD/GCE o-Phenylene Diamine/Glassy Carbon Electrode

PDDAC Poly-(diallyldimethylammonium chloride)

POM Polyoxometalate

rGO Reduced Graphene Oxide

SWV Square Wave Voltammetry

The performance of electrochemical measurements is strongly influenced by the material of the working electrode. For many years, mercury working electrodes such as the hanging mercury drop electrode and mercury film electrode were the electrodes of choice due to the highly reproducible, renewable, and smooth surface of mercury [104]. However, these electrodes become increasingly disfavoured and banned in some countries due to health and environmental considerations. Alternative, environmentally friendly electrodes and chemically modified electrodes have thus become the focus of substantial recent research studies and have received great attention in electroanalysis due to their low background current, wide potential window, chemical inertness, low cost, and suitability for various sensing and detection applications [105] Where the most popular schemes used to substitute mercury electrodes include boron-doped diamond electrodes [97], carbon nanotube electrodes [99, 106], and screen printed electrodes [26].

Electrochemical methods have advantages in that they generally minimize hazardous substance usage and waste generation during analyses.

\subsection{Capillary Zone Electrophoresis Methods}

A number of sample extraction and pre-concentration methods for triclosan have been mentioned for the various analytical techniques discussed in this paper. Similarly a number of extraction methods have been used in conjunction with capillary zone electrophoresis for the determination of triclosan. The combined use of dispersive liquid-liquid microextraction with capillary zone electrophoresis-UV detection (CZE-UV), for example, can be applied to detect trace amounts of triclosan in different matrices. This technique provides the considerable advantage of little matrix interference, good repeatability and recoveries within a short time and relatively high enrichment factors [58].

A rapid and convenient method was developed for the simultaneous determination of triclosan and bisphenol A (BPA) in water samples by dispersive liquid-liquid microextraction (DLLME) combined with capillary zone electrophoresis with UV detection (CZE-UV) [58]. Under optimized 
conditions, the calibration curve for triclosan was linear over the range of $2.0 \times 10^{4}-2.0 \times 10^{6} \mathrm{ng} \mathrm{L}^{-1}$ with a detection limit of $4.0 \times 10^{3} \mathrm{ng} \mathrm{L}^{-1}$.

A sensitive method was also developed using capillary zone electrophoresis with UV-diode array detection (CZE-DAD) for simultaneous determination of 19 pharmaceutical and personal care products, including triclosan, in wastewater. Solid phase extraction in combination with online pre-concentration methodology was applied. The proposed method provided the linear range of $1.0 \times 10^{5}-1.0 \times 10^{6} \mathrm{ng} \mathrm{L}^{-1}$ with a detection limit of $30.6 \mathrm{ng} \mathrm{L}^{-1}$ for triclosan [107].

Another study was conducted for the determination of triclosan in untreated wastewater by capillary electrophoresis (CE) with UV detection. Instrument linear range, detection and quantification limits were $2.4 \times 10^{6}-3.8 \times 10^{7}, 1.2 \times 10^{6}$ and $2.4 \times 10^{6} \mathrm{ng} \mathrm{L}^{-1}$ respectively, with a method detection limit of $68.7 \times 10^{3} \mathrm{ng} \mathrm{L}^{-1}[59]$.

There are limited studies regarding the application of capillary zone electrophoresis for the determination of triclosan in water samples. Despite the advantages of $\mathrm{CE}$, including reduced reagent consumption, low required sample volume, high separation efficiency and short analysis time; it provides low sensitivity in terms of solute concentration compared to other separation techniques, which is due to both the small optical path-length of the capillary $(50-75 \mu \mathrm{m})$ used as a detection cell and the small volumes (usually a few nanoliters) that can be injected, which limit the application of CE $[108,109]$. Furthermore, the main drawbacks of CZE compared to other analytical methods are its relatively poor reproducibility and low sample tolerance [110]. The disadvantage of CE with regard to its inferior reproducibility as compared to HPLC is such an obstacle that HPLC is preferred for routine applications [111]. A certain amount of avoidance can be explained by the lack of familiarity with CE which can lead to the development of methods that are not robust, perform poorly and give CE a negative reputation [111]. Table 5 compares some analytical parameters of HPLC and CZE.

Table 5. Comparison of typical analytical parameters pertaining to liquid chromatography and capillary electrophoresis [112].

\begin{tabular}{|c|c|c|}
\hline Parameter & HPLC & CE \\
\hline Injected volume & $1-100 \mu \mathrm{L}$ & $1-100 \mathrm{~nL}$ \\
\hline Flow rate of the liquid phase & $1-10 \mathrm{~mL} / \mathrm{min}$ & $1-100 \mathrm{~nL} / \mathrm{min}$ \\
\hline
\end{tabular}




\begin{tabular}{|c|c|c|}
\hline Analysis time & 10-60 min & 1-20 min \\
\hline Separation technique & $\begin{array}{c}\text { High pressure with complicated } \\
\text { pumping system }\end{array}$ & $\begin{array}{c}\text { Electrical field with stable high } \\
\text { voltage source, no back } \\
\text { pressure }\end{array}$ \\
\hline Solvents & Different solvents for different \\
& columns & $\begin{array}{c}\text { Different solvents in the same } \\
\text { column }\end{array}$ \\
\hline
\end{tabular}

\subsection{Optical Methods}

An ultrasound-assisted emulsification-microextraction procedure for triclosan determination in wastewater samples was developed wherea diazotation reaction was employed with microvolume UV-Vis spectrophotometry detection. A linear range of 5.8-52.1 $\mathrm{ng} \mathrm{L}^{-1}$ was obtained and the LOD was $1.45 \mathrm{ng} \mathrm{L}^{-1}$ [113].

White light reflectance spectroscopy (WLRS) has also been used for the real-time monitoring of triclosan using synthesized 2,3-dimethyl-6-(undec-10-enamide)-6-deoxy- $\beta$-cyclodextrin (DMBUA) on a silicon wafer coated with a novolac resin, where the introduction of triclosan on the top of the DMBUA layer resulted in a peak wavelength shift [114].

Although spectrophotometric methods are comparatively cheap and fast, they are not as sensitive as the other methods described, hence they are generally not suitable for the direct monitoring of triclosan in environmental water samples, as analyte pre-concentration would be required. Alternative optical methods which are more sensitive, such as surface plasmon resonance based techniques have thus been developed.

In spite of the fact that chemiluminescence methods are convenient, fast and require less expensive instrumentation, pre-transformation is needed prior to triclosan analysis and the selectivity achieved is generally not good enough for direct application in water analysis [57]. Therefore there still remains a great need for a fast and user-friendly in situ monitoring device for triclosan.

A TCS-imprinted poly(2-hydroxyethylmethacrylate-methacryloylamidoglutamic acid) [p(HEMAGA)] surface plasmon resonance (SPR) nanosensor was developed as a novel molecularly imprinted SPR sensor for the sensitive and selective detection of triclosan in wastewater, using an allylmercaptane modified gold SPR chip and imprinted p(HEMAGA) nanofilm. The linearity range and detection limit of TCS were found to be $50-1.0 \times 10^{3} \mathrm{ng} \mathrm{L}^{-1}$ and $17 \mathrm{ng} \mathrm{L}^{-1}$, respectively with a LOQ of $50 \mathrm{ng} \mathrm{L}^{-1}[115]$. 
It is noteworthy to mention that, with the advent of mathematical data treatment (chemometrics), solvent-free methodologies based on direct spectroscopic measurements without any sample pretreatment have been developed for a range of analytes to provide quantitative as well as qualitative information [93]. These advances may result in interesting possibilities regarding the future establishment of both screening and quantitative spectroscopic methods.

\section{Conclusion and future outlook}

Triclosan is a synthetic, non-ionic, broad-spectrum antimicrobial agent that has been used extensively for more than 20 years. It is a relatively stable, lipophilic compound which has been detected in surface water systems. In general, due to the low concentration of triclosan in environmental matrices, it needs to be extracted and pre-concentrated before analysis. Traditional extraction techniques are not environmentally friendly and consumption of a large quantity of chemicals limits their application, therefore alternatives such as dispersive liquidliquid microextraction has gained considerable attention, as it does not only provide better efficiency but is also a time-saving process because of the large contact surface involved and thus high speed of analyte mass transfer.

We have reviewed different approaches for the determination of triclosan and their linear ranges and detection limits have been compared. The determination of triclosan has predominately been performed by chromatography based methods and nanomaterial based electrode systems using voltammetric methods. Chromatography methods are sensitive and selective, however they are time consuming and they require bulky and expensive instrumentation, as well as high cost detectors such as MS and MS/MS. Triclosan can undergo oxidation at the surface of electrode materials, making it suitable for electrochemical detection. The combination of nanomaterials and electrochemical methods provide a fast, convenient and inexpensive approach for the determination of triclosan, however these methods suffer from strongly adsorbed oligomeric products of the oxidation of triclosan on the electrode surface.

Relatively few reports have been published recently on CE developments for the determination of triclosan. However, if reproducibility limitations of CE can be adequately addressed, green analytical chemistry requirements might lead to CE becoming a viable alternative to HPLC in the future in some applications [116].

Although spectrophotometric methods enjoy simplicity, and a wide linear range, they cannot be applied for direct application in water samples. The complexity of matrices also limits the application of spectroscopic methods in addition to their low sensitivity, which results in the need 
for clean-up and pre-concentration procedures. The relatively new development of employing molecularly imprinted polymers in the extraction and sensing of triclosan is a promising contribution for the determination of triclosan in water samples, due to the enhanced sensitivity and selectivity which can result, as well as due to the low cost and mechanical stability of these materials.

Although GC or LC with MS is the method which is primarily used in routine applications, other techniques reported in this review are being developed and may see wider application once they become more widely known and tested. In many cases quality control needs further attention and analytical robustness needs to be proven following from the reporting of initial development results, to allow for the uptake of these methods for wider and routine applications. Due to the ubiquity of triclosan in the environment, we expect these developments to be realized for those methods showing promising selectivity and sensitivity, such as the application of MIPs. Regarding GAC, online, miniaturized and automated sampling and analytical approaches are favourable, where solvent consumption is minimized. Some routine methods based on classical chromatographic analyses consume large amount of reagents or energy and they also generate large volumes of waste. These methods therefore require improvements so that they become more environmentally friendly.

As a result of the ubiquitous nature of triclosan in environmental water resources and due the concern regarding its potential environmental impacts, it is envisaged that future research efforts will focus on the development of novel methods which provide for the fast, simple, sensitive and selective determination of this analyte in a cost effective and environmentally responsible manner.

\section{Acknowledgements}

Funding from the University of Pretoria, the Water Research Commission (grant K5-2438), the Photonics Initiative of South Africa (grant PISA-15-DIR-06) and the National Research Foundation of South Africa (grants 90720 and 93394) is gratefully acknowledged.

\section{References}

[1] H. Bhargava, P.A. Leonard, Triclosan: applications and safety, American Journal of Infection Control, 24 (1996) 209-218. 
[2] R.D. Jones, H.B. Jampani, J.L. Newman, A.S. Lee, Triclosan: a review of effectiveness and safety in health care settings, American Journal of Infection Control, 28 (2000) 184-196.

[3] J.V. Rodricks, J.A. Swenberg, J.F. Borzelleca, R.R. Maronpot, A.M. Shipp, Triclosan: a critical review of the experimental data and development of margins of safety for consumer products, Critical Reviews in Toxicology, 40 (2010) 422-484.

[4] EU, Cosmetics Directive 76/768//EEC, Annex VI, Part I, entry 25, List of Preservatives which cosmetic products may contain 2007.

[5] J. Du Preez, W. Yang, Improving the aqueous solubility of triclosan by solubilization, complexation, and in situ salt formation, Journal of Cosmetic Science, 54 (2003) 537-550.

[6] H.-S. Son, G. Ko, K.-D. Zoh, Kinetics and mechanism of photolysis and TiO2 photocatalysis of triclosan, Journal of Hazardous Materials, 166 (2009) 954-960.

[7] M. Allmyr, F. Harden, L.-M.L. Toms, J.F. Mueller, M.S. McLachlan, M. Adolfsson-Erici, et al., The influence of age and gender on triclosan concentrations in Australian human blood serum, Science of the total environment, 393 (2008) 162-167.

[8] N. Veldhoen, R.C. Skirrow, H. Osachoff, H. Wigmore, D.J. Clapson, M.P. Gunderson, et al., The bactericidal agent triclosan modulates thyroid hormone-associated gene expression and disrupts postembryonic anuran development, Aquatic Toxicology, 80 (2006) 217-227.

[9] J.-L. Zhao, Q.-Q. Zhang, F. Chen, L. Wang, G.-G. Ying, Y.-S. Liu, et al., Evaluation of triclosan and triclocarban at river basin scale using monitoring and modeling tools: implications for controlling of urban domestic sewage discharge, Water Research, 47 (2013) 395-405.

[10] N. Tatarazako, H. Ishibashi, K. Teshima, K. Kishi, K. Arizono, Effects of triclosan on various aquatic organisms, Environmental sciences: an international journal of environmental physiology and toxicology, DOI (2004) 133-140.

[11] M. Allmyr, M. Adolfsson-Erici, M.S. McLachlan, G. Sandborgh-Englund, Triclosan in plasma and milk from Swedish nursing mothers and their exposure via personal care products, Science of the Total Environment, 372 (2006) 87-93.

[12] E.M. Fiss, K.L. Rule, P.J. Vikesland, Formation of chloroform and other chlorinated byproducts by chlorination of triclosan-containing antibacterial products, Environmental science \& technology, 41 (2007) 2387-2394.

[13] K.L. Rule, V.R. Ebbett, P.J. Vikesland, Formation of chloroform and chlorinated organics by free-chlorine-mediated oxidation of triclosan, Environmental Science \& Technology, 39 (2005) 3176-3185. 
[14] A. Kanetoshi, H. Ogawa, E. Katsura, H. Kaneshima, Chlorination of Irgasan DP300 and formation of dioxins from its chlorinated derivatives, Journal of Chromatography A, 389 (1987) 139-153.

[15] A. Lindström, I.J. Buerge, T. Poiger, P.-A. Bergqvist, M.D. Muller, H.-R. Buser, Occurrence and Environmental Behavior of the Bactericide Triclosan and Its Methyl Derivative in Surface Waters and in Wastewater, Environmental Science and Technology, 36 (2002) 2322-2329.

[16] M.M. Häggblom, D. Janke, P.J. Middeldorp, M.S. Salkinoja-Salonen, O-methylation of chlorinated phenols in the genus Rhodococcus, Archives of microbiology, 152 (1989) 6-9.

[17] D.W. Kolpin, E.T. Furlong, M.T. Meyer, E.M. Thurman, S.D. Zaugg, L.B. Barber, et al., Pharmaceuticals, hormones, and other organic wastewater contaminants in US streams, 19992000: A national reconnaissance, Environmental Science \& Technology, 36 (2002) 1202-1211.

[18] G.S. Dhillon, S. Kaur, R. Pulicharla, S.K. Brar, M. Cledón, M. Verma, et al., Triclosan: current status, occurrence, environmental risks and bioaccumulation potential, International journal of environmental research and public health, 12 (2015) 5657-5684.

[19] SCCS, Opinion on triclosan - antimicrobial resistance, Scientific Committee on Consumer Safety, 2010, pp. 1-56.

[20] T.E. Chalew, R.U. Halden, Environmental exposure of aquatic and terrestrial biota to triclosan and triclocarban, Journal of the American Water Resources Association, 45 (2009) 413.

[21] K. Bester, Fate of triclosan and triclosan-methyl in sewage treatmentplants and surface waters, Archives of Environmental Contamination and Toxicology, 49 (2005) 9-17.

[22] M. Parolini, A. Pedriali, A. Binelli, Application of a biomarker response index for ranking the toxicity of five pharmaceutical and personal care products (PPCPs) to the bivalve Dreissena polymorpha, Archives of environmental contamination and toxicology, 64 (2013) 439-447.

[23] S.A.O. de García, G.P. Pinto, P.A. García-Encina, R. Irusta-Mata, Ecotoxicity and environmental risk assessment of pharmaceuticals and personal care products in aquatic environments and wastewater treatment plants, Ecotoxicology, 23 (2014) 1517-1533.

[24] S.O. de García, P. García-Encina, R. Irusta-Mata, Environmental risk assessment (ERA) of pharmaceuticals and personal care products (PPCPs) using ecotoxicity tests, DOI (2011).

[25] USEPA, Targeted national sewage sludge survey sampling and analysis technical report, United States Environmental Protection Agency Washington, DC, 2009, pp. 1-10.

[26] R.M. Pemberton, J.P. Hart, Electrochemical behaviour of triclosan at a screen-printed carbon electrode and its voltammetric determination in toothpaste and mouthrinse products, Analytica Chimica Acta, 390 (1999) 107-115. 
[27] D. Mackay, R.S. Boethling, Handbook of property estimation methods for chemicals: environmental health sciences, CRC press2000.

[28] B. List, Reregistration Eligibility Decision for Triclosan, DOI (2008).

[29] E. Aldous, L. Rockett, I. Johnson, Proposed EQS for Water Framework Directive Annex VIII Substances: Triclosan (For Consultation), Water Framework Directive-United Kingdom Technical Advisory Group, DOI (2009).

[30] D.C. McAvoy, B. Schatowitz, M. Jacob, A. Hauk, W.S. Eckhoff, Measurement of triclosan in wastewater treatment systems, Environmental Toxicology and Chemistry, 21 (2002) 1323-1329. [31] NICNAS, Triclosan, Priority Existing Chemical Assessment Report No. 30, National Industrial Chemicals Notification and Assessment, Sydney, Australia, 2009.

[32] R. Kanda, P. Griffin, H.A. James, J. Fothergill, Pharmaceutical and personal care products in sewage treatment works, Journal of Environmental Monitoring, 5 (2003) 823-830.

[33] K. Bester, Triclosan in a sewage treatment process-balances and monitoring data, Water Research, 37 (2003) 3891-3896.

[34] H. Singer, S. Müller, C. Tixier, L. Pillonel, Triclosan: occurrence and fate of a widely used biocide in the aquatic environment: field measurements in wastewater treatment plants, surface waters, and lake sediments, Environmental Science \& Technology, 36 (2002) 4998-5004.

[35] T.W. Federle, S.K. Kaiser, B.A. Nuck, Fate and effects of triclosan in activated sludge, Environmental Toxicology and Chemistry, 21 (2002) 1330-1337.

[36] L. Lishman, S.A. Smyth, K. Sarafin, S. Kleywegt, J. Toito, T. Peart, et al., Occurrence and reductions of pharmaceuticals and personal care products and estrogens by municipal wastewater treatment plants in Ontario, Canada, Science of the Total Environment, 367 (2006) 544-558.

[37] A. Lindström, I.J. Buerge, T. Poiger, P.-A. Bergqvist, M.D. Müller, H.-R. Buser, Occurrence and environmental behavior of the bactericide triclosan and its methyl derivative in surface waters and in wastewater, Environmental science \& technology, 36 (2002) 2322-2329.

[38] V. Lopez-Avila, R.A. Hites, Organic compounds in an industrial wastewater. Their transport into sediments, Environmental Science \& Technology, 14 (1980) 1382-1390.

[39] A. Thompson, P. Griffin, R. Stuetz, E. Cartmell, The fate and removal of triclosan during wastewater treatment, Water environment research, DOI (2005) 63-67.

[40] P. Canosa, S. Morales, I. Rodriguez, E. Rubi, R. Cela, M. Gomez, Aquatic degradation of triclosan and formation of toxic chlorophenols in presence of low concentrations of free chlorine, Analytical and bioanalytical chemistry, 383 (2005) 1119-1126. 
[41] S. Suarez, M.C. Dodd, F. Omil, U. von Gunten, Kinetics of triclosan oxidation by aqueous ozone and consequent loss of antibacterial activity: relevance to municipal wastewater ozonation, Water Res, 41 (2007) 2481-2490.

[42] E.C. Wert, F.L. Rosario-Ortiz, S.A. Snyder, Effect of ozone exposure on the oxidation of trace organic contaminants in wastewater, Water Research, 43 (2009) 1005-1014.

[43] M.C. Dodd, H.-P.E. Kohler, U. Von Gunten, Oxidation of antibacterial compounds by ozone and hydroxyl radical: elimination of biological activity during aqueous ozonation processes, Environmental Science \& Technology, 43 (2009) 2498-2504.

[44] S. Snyder, E. Wert, H. Lei, P. Westerhoff, Y. Yoon, Removal of EDCs and pharmaceuticals in drinking and reuse treatment processes (91188), Denver, CO, USA: American Water Works Association Research Foundation (AWWARF), DOI (2007).

[45] G.F. Lee, J.C. Morris, Kinetics of chlorination of phenol-chlorophenolic tastes and odors, Int. J. Air Water Pollut, 6 (1962) 419-431.

[46] C. Tixier, H.P. Singer, S. Canonica, S.R. Müller, Phototransformation of triclosan in surface waters: A relevant elimination process for this widely used biocide laboratory studies, field measurements, and modeling, Environmental science \& technology, 36 (2002) 3482-3489.

[47] J. Namieśnik, Green analytical chemistry-some remarks, Journal of Separation Science, 24 (2001) 151-153.

[48] A. Gałuszka, Z. Migaszewski, J. Namieśnik, The 12 principles of green analytical chemistry and the SIGNIFICANCE mnemonic of green analytical practices, TrAC Trends in Analytical Chemistry, 50 (2013) 78-84.

[49] D. Kim, J. Han, Y. Choi, On-line solid-phase microextraction of triclosan, bisphenol A, chlorophenols, and selected pharmaceuticals in environmental water samples by highperformance liquid chromatography-ultraviolet detection, Analytical and Bioanalytical Chemistry, 405 (2013) 377-387.

[50] C. Zheng, J. Zhao, P. Bao, J. Gao, J. He, Dispersive liquid-liquid microextraction based on solidification of floating organic droplet followed by high-performance liquid chromatography with ultraviolet detection and liquid chromatography-tandem mass spectrometry for the determination of triclosan and 2, 4-dichlorophenol in water samples, Journal of Chromatography A, 1218 (2011) 3830-3836.

[51] R. Gibson, E. Becerril-Bravo, V. Silva-Castro, B. Jiménez, Determination of acidic pharmaceuticals and potential endocrine disrupting compounds in wastewaters and spring waters by selective elution and analysis by gas chromatography-mass spectrometry, Journal of Chromatography A, 1169 (2007) 31-39. 
[52] Z. Yu, S. Peldszus, P.M. Huck, Optimizing gas chromatographic-mass spectrometric analysis of selected pharmaceuticals and endocrine-disrupting substances in water using factorial experimental design, Journal of Chromatography A, 1148 (2007) 65-77.

[53] H. Lu, H. Ma, G. Tao, Spectrophotometric determination of triclosan in personal care products, Spectrochimica Acta Part A: Molecular and Biomolecular Spectroscopy, 73 (2009) 854-857.

[54] L.H.-H.T. Guan-Hong, Spectrophotometric Determination of Triclosan in Personal Care Products, Chinese Journal of Spectroscopy Laboratory, 3 (2009) 013.

[55] M. Amiri, S. Shahrokhian, E. Psillakis, F. Marken, Electrostatic accumulation and determination of triclosan in ultrathin carbon nanoparticle composite film electrodes, Analytica Chimica Acta, 593 (2007) 117-122.

[56] M.L. Yola, N. Atar, H. Karimi-Maleh, S. Wang, Sensitive and selective determination of aqueous triclosan based on gold nanoparticles on polyoxometalate/reduced graphene oxide nanohybrid, RSC Advances, 5 (2015) 65953-65962.

[57] Y. Liu, Q.-J. Song, L. Wang, Development and characterization of an amperometric sensor for triclosan detection based on electropolymerized molecularly imprinted polymer, Microchemical Journal, 91 (2009) 222-226.

[58] H. Wang, A. Zhang, W. Wang, M. Zhang, H. Liu, X. Wang, Separation and Determination of Triclosan and Bisphenol A in Water, Beverage, and Urine Samples by Dispersive Liquid-Liquid Microextraction Combined with Capillary Zone Electrophoresis-UV Detection, Journal of AOAC International, 96 (2013) 459-465.

[59] S.E. Gibbons, C. Wang, Y. Ma, Determination of pharmaceutical and personal care products in wastewater by capillary electrophoresis with UV detection, Talanta, 84 (2011) 11631168.

[60] M. Guardia, S. Armenta, Green solvents for analytical separation and analyses, Encyclopedia of Analytical Chemistry, DOI (2010).

[61] P. Sun, D.W. Armstrong, lonic liquids in analytical chemistry, Analytica Chimica Acta, 661 (2010) 1-16.

[62] M. Tobiszewski, J. Namiesnik, Developments in green chromatography, LC GC EUROPE, 27 (2014) 405-408.

[63] U.S. EPA, Method 1694: Pharmaceuticals and Personal Care Products in Water, Soil, Sediment, and Biosolids by HPLC/MS/MS, 2007, pp. 1-77. 
[64] J.C. Chow, J.G. Watson, J. Robles, X. Wang, L.-W.A. Chen, D.L. Trimble, et al., Quality assurance and quality control for thermal/optical analysis of aerosol samples for organic and elemental carbon, Analytical and Bioanalytical Chemistry, 401 (2011) 3141-3152.

[65] I. Taverniers, M. De Loose, E. Van Bockstaele, Trends in quality in the analytical laboratory. II. Analytical method validation and quality assurance, TrAC Trends in Analytical Chemistry, 23 (2004) 535-552.

[66] J.-H. Guo, X.-H. Li, X.-L. Cao, Y. Li, X.-Z. Wang, X.-B. Xu, Determination of triclosan, triclocarban and methyl-triclosan in aqueous samples by dispersive liquid-liquid microextraction combined with rapid liquid chromatography, Journal of Chromatography A, 1216 (2009) 30383043.

[67] G. Gatidou, N.S. Thomaidis, A.S. Stasinakis, T.D. Lekkas, Simultaneous determination of the endocrine disrupting compounds nonylphenol, nonylphenol ethoxylates, triclosan and bisphenol $A$ in wastewater and sewage sludge by gas chromatography-mass spectrometry, Journal of Chromatography A, 1138 (2007) 32-41.

[68] M.G. Pintado-Herrera, E. González-Mazo, P.A. Lara-Martín, Determining the distribution of triclosan and methyl triclosan in estuarine settings, Chemosphere, 95 (2014) 478-485.

[69] L. Jachero, B. Sepúlveda, I. Ahumada, E. Fuentes, P. Richter, Rotating disk sorptive extraction of triclosan and methyl-triclosan from water samples, Analytical and bioanalytical chemistry, 405 (2013) 7711-7716.

[70] Y.C. Fiamegos, C.D. Stalikas, In-drop derivatisation liquid-phase microextraction assisted by ion-pairing transfer for the gas chromatographic determination of phenolic endocrine disruptors, Analytica Chimica Acta, 597 (2007) 32-40.

[71] M. Kawaguchi, R. Ito, H. Honda, N. Endo, N. Okanouchi, K. Saito, et al., Stir bar sorptive extraction and thermal desorption-gas chromatography-mass spectrometry for trace analysis of triclosan in water sample, Journal of Chromatography A, 1206 (2008) 196-199.

[72] A.M.C. Ferreira, M. Möder, M.E.F. Laespada, GC-MS determination of parabens, triclosan and methyl triclosan in water by in situ derivatisation and stir-bar sorptive extraction, Analytical and bioanalytical chemistry, 399 (2011) 945-953.

[73] I. González-Mariño, J.B. Quintana, I. Rodríguez, S. Schrader, M. Moeder, Fully automated determination of parabens, triclosan and methyl triclosan in wastewater by microextraction by packed sorbents and gas chromatography-mass spectrometry, Analytica chimica acta, 684 (2011) 59-66.

[74] J.-L. Wu, N.P. Lam, D. Martens, A. Kettrup, Z. Cai, Triclosan determination in water related to wastewater treatment, Talanta, 72 (2007) 1650-1654. 
[75] M. Gómez, A. Agüera, M. Mezcua, J. Hurtado, F. Mocholí, A. Fernández-Alba, Simultaneous analysis of neutral and acidic pharmaceuticals as well as related compounds by gas chromatography-tandem mass spectrometry in wastewater, Talanta, 73 (2007) 314-320.

[76] W.C. Chau, J.L. Wu, Z. Cai, Investigation of levels and fate of triclosan in environmental waters from the analysis of gas chromatography coupled with ion trap mass spectrometry, Chemosphere, 73 (2008) S13-17.

[77] J. Regueiro, E. Becerril, C. Garcia-Jares, M. Llompart, Trace analysis of parabens, triclosan and related chlorophenols in water by headspace solid-phase microextraction with in situ derivatization and gas chromatography-tandem mass spectrometry, Journal of Chromatography A, 1216 (2009) 4693-4702.

[78] R. Montes, I. Rodríguez, E. Rubí, R. Cela, Dispersive liquid-liquid microextraction applied to the simultaneous derivatization and concentration of triclosan and methyltriclosan in water samples, Journal of Chromatography A, 1216 (2009) 205-210.

[79] J. Regueiro, M. Llompart, E. Psillakis, J.C. Garcia-Monteagudo, C. Garcia-Jares, Ultrasound-assisted emulsification-microextraction of phenolic preservatives in water, Talanta, 79 (2009) 1387-1397.

[80] E. Villaverde-de-Sáa, I. González-Mariño, J.B. Quintana, R. Rodil, I. Rodríguez, R. Cela, Insample acetylation-non-porous membrane-assisted liquid-liquid extraction for the determination of parabens and triclosan in water samples, Analytical and bioanalytical chemistry, 397 (2010) 2559-2568.

[81] A.R.M. Silva, J. Nogueira, New approach on trace analysis of triclosan in personal care products, biological and environmental matrices, Talanta, 74 (2008) 1498-1504.

[82] J.Y. Shen, M.S. Chang, S.H. Yang, G.J. Wu, Simultaneous determination of triclosan, triclocarban, and transformation products of triclocarban in aqueous samples using solid-phase micro-extraction-HPLC-MS/MS, Journal of Separation Science, 35 (2012) 2544-2552.

[83] M.S. Chang, J.Y. Shen, S.-H. Yang, G.J. Wu, Determination of three endocrine disrupting chemicals, triclosan, 4-n-nonylphenol, and di-n-butyl phthalate using stir bar sorptive extraction in samples of different matrices, Toxicological \& Environmental Chemistry, 94 (2012) 1027 1033.

[84] E. Carmona, V. Andreu, Y. Picó, Occurrence of acidic pharmaceuticals and personal care products in Turia River Basin: from waste to drinking water, Science of the total environment, 484 (2014) 53-63.

[85] R.-S. Zhao, X. Wang, J. Sun, S.-S. Wang, J.-P. Yuan, X.-K. Wang, Trace determination of triclosan and triclocarban in environmental water samples with ionic liquid dispersive liquid- 
phase microextraction prior to HPLC-ESI-MS-MS, Analytical and bioanalytical chemistry, 397 (2010) 1627-1633.

[86] R.S. Zhao, X. Wang, J. Sun, J.P. Yuan, S.S. Wang, X.K. Wang, Temperature-controlled ionic liquid dispersive liquid-phase microextraction for the sensitive determination of triclosan and triclocarban in environmental water samples prior to HPLC-ESI-MS/MS, Journal of separation science, 33 (2010) 1842-1848.

[87] R.-S. Zhao, X. Wang, J. Sun, C. Hu, X.-K. Wang, Determination of triclosan and triclocarban in environmental water samples with ionic liquid/ionic liquid dispersive liquid-liquid microextraction prior to HPLC-ESI-MS/MS, Microchimica Acta, 174 (2011) 145-151.

[88] J. Sun, C.L. Yi, R.S. Zhao, X. Wang, W.Q. Jiang, X.K. Wang, Determination of trace triclosan in environmental water by microporous bamboo-activated charcoal solid-phase extraction combined with HPLC-ESI-MS, Journal of separation science, 35 (2012) 2781-2786.

[89] J. Guo, X. Li, X. Cao, L. Qu, D. Hou, X. Xu, Temperature-controlled ionic liquid dispersive liquid phase microextraction combined with ultra-high-pressure liquid chromatography for the rapid determination of triclosan, triclocarban and methyl-triclosan in aqueous samples, Science China Chemistry, 53 (2010) 2600-2607.

[90] M. Pedrouzo, F. Borrull, R.M. Marcé, E. Pocurull, Ultra-high-performance liquid chromatography-tandem mass spectrometry for determining the presence of eleven personal care products in surface and wastewaters, Journal of Chromatography A, 1216 (2009) 69947000.

[91] R. Gao, X. Kong, F. Su, X. He, L. Chen, Y. Zhang, Synthesis and evaluation of molecularly imprinted core-shell carbon nanotubes for the determination of triclosan in environmental water samples, Journal of Chromatography A, 1217 (2010) 8095-8102.

[92] J. Płotka, M. Tobiszewski, A.M. Sulej, M. Kupska, T. Gorecki, J. Namieśnik, Green chromatography, Journal of Chromatography A, 1307 (2013) 1-20.

[93] S. Armenta, M. De la Guardia, Green Spectroscopy: A scientometric picture, Spectroscopy Letters, 42 (2009) 277-283.

[94] L. Kocúrová, I.S. Balogh, V. Andruch, Solvent microextraction: A review of recent efforts at automation, Microchemical Journal, 110 (2013) 599-607.

[95] H. Dai, G. Xu, L. Gong, C. Yang, Y. Lin, Y. Tong, et al., Electrochemical detection of triclosan at a glassy carbon electrode modifies with carbon nanodots and chitosan, Electrochimica Acta, 80 (2012) 362-367.

[96] A. Safavi, N. Maleki, H. Shahbaazi, Electrochemical determination of triclosan at a mercury electrode, Analytica chimica acta, 494 (2003) 225-233. 
[97] M.A. Ghanem, R.G. Compton, B.A. Coles, E. Psillakis, M.A. Kulandainathan, F. Marken, Microwave activation of electrochemical processes: High temperature phenol and triclosan electro-oxidation at carbon and diamond electrodes, Electrochimica Acta, 53 (2007) 1092-1099. [98] M.A. Ghanem, R.G. Compton, B.A. Coles, A. Canals, F. Marken, Microwave enhanced electroanalysis of formulations: processes in micellar media at glassy carbon and at platinum electrodes, Analyst, 130 (2005) 1425-1431.

[99] M. Moyo, L.R. Florence, J.O. Okonkwo, Improved electro-oxidation of triclosan at nano-zinc oxide-multiwalled carbon nanotube modified glassy carbon electrode, Sensors and Actuators B: Chemical, 209 (2015) 898-905.

[100] L. Vidal, A. Chisvert, A. Canals, E. Psillakis, A. Lapkin, F. Acosta, et al., Chemically surface-modified carbon nanoparticle carrier for phenolic pollutants: Extraction and electrochemical determination of benzophenone- 3 and triclosan, Analytica Chimica Acta, 616 (2008) 28-35.

[101] M.J. Bonne, K.J. Edler, J.G. Buchanan, D. Wolverson, E. Psillakis, M. Helton, et al., Thinfilm modified electrodes with reconstituted cellulose-PDDAC films for the accumulation and detection of triclosan, The Journal of Physical Chemistry C, 112 (2008) 2660-2666.

[102] L. Fotouhi, H.R. Shahbaazi, A. Fatehi, M.M. Heravi, Voltammetric determination of triclosan in waste water and personal care products, Int J Electrochem Sci, 5 (2010) 1390-1398. [103] B. Li, Z. Qiu, Q. Wan, Y. Liu, N. Yang, $\beta$-cyclodextrin functionalized graphene nano platelets for electrochemical determination of triclosan, Physica Status Solidi (A), 211 (2014) 2773-2777.

[104] A.H. Alghamdi, Applications of stripping voltammetric techniques in food analysis, Arabian Journal of Chemistry, 3 (2010) 1-7.

[105] J. Wang, Real-time electrochemical monitoring: toward green analytical chemistry, Accounts of Chemical Research, 35 (2002) 811-816.

[106] J. Yang, P. Wang, X. Zhang, K. Wu, Electrochemical sensor for rapid detection of Triclosan using a multiwall carbon nanotube film, Journal of Agricultural and Food Chemistry, 57 (2009) 9403-9407.

[107] Z. Zhang, D. Zhang, X. Zhang, Simultaneous determination of pharmaceutical and personal care products in wastewater by capillary electrophoresis with head-column fieldamplified sample stacking, Analytical Methods, 6 (2014) 7978-7983.

[108] F.J. Lara, D. Airado-Rodríguez, D. Moreno-González, J.F. Huertas-Pérez, A.M. GarcíaCampaña, Applications of capillary electrophoresis with chemiluminescence detection in clinical, environmental and food analysis. A review, Analytica Chimica Acta, 913 (2016) 22-40. 
[109] D.M. Osbourn, D.J. Weiss, C.E. Lunte, On-line preconcentration methods for capillary electrophoresis, Electrophoresis, 21 (2000) 2768.

[110] J. Frenz, S.-L. Wu, W.S. Hancock, Characterization of human growth hormone by capillary electrophoresis, Journal of Chromatography A, 480 (1989) 379-391.

[111] M. de la Guardia, S. Garrigues, Handbook of green analytical chemistry, Wiley Online Library2012.

[112] M. Koel, M. Kaljurand, Application of the principles of green chemistry in analytical chemistry, Pure and applied chemistry, 78 (2006) 1993-2002.

[113] N. Cabaleiro, F. Pena-Pereira, I. de la Calle, C. Bendicho, I. Lavilla, Determination of triclosan by cuvetteless UV-vis micro-spectrophotometry following simultaneous ultrasound assisted emulsification-microextraction with derivatization: Use of a micellar-ionic liquid as extractant, Microchemical Journal, 99 (2011) 246-251.

[114] D. Maffeo, Z. Velkov, K. Misiakos, K. Mergia, A. Paulidou, M. Zavali, et al., Real-time monitoring of nanomolar binding to a cyclodextrin monolayer immobilized on a Si/SiO 2/novolac surface using white light reflectance spectroscopy: The case of triclosan, Journal of colloid and interface science, 358 (2011) 369-375.

[115] N. Atar, T. Eren, M.L. Yola, S. Wang, A sensitive molecular imprinted surface plasmon resonance nanosensor for selective determination of trace triclosan in wastewater, Sensors and Actuators B: Chemical, 216 (2015) 638-644.

[116] K.D. Altria, W. Ciccone, B. Nunnally, Capillary Electrophoresis, LC-GC Technology forum, 2009. 